\title{
Statistical approaches to lifetime measurements with restricted observation times
}

\author{
X. C. Chen, ${ }^{1, *}$ Q. Zeng, ${ }^{1,2}$ Yu. A. Litvinov, ${ }^{1,3,4}$ X. L. Tu, ${ }^{1,4}$ P. M. Walker, ${ }^{5}$ M. Wang, ${ }_{1}^{1}$ Q. Wang, ${ }^{1,6}$ K. Yue, ${ }^{1}$ and Y. H. Zhang ${ }^{1}$ \\ ${ }^{1}$ Institute of Modern Physics, Chinese Academy of Sciences, Lanzhou 730000, China \\ ${ }^{2}$ University of Science and Technology of China, Hefei 230026, China \\ ${ }^{3}$ GSI Helmholtzzentrum für Schwerionenforschung, Darmstadt 64291, Germany \\ ${ }^{4}$ Max-Planck-Institut für Kernphysik, Heidelberg 69117, Germany \\ ${ }^{5}$ Department of Physics, University of Surrey, Guildford GU2 7XH, United Kingdom \\ ${ }^{6}$ University of Chinese Academy of Sciences, Beijing 100049, China
}

(Received 14 June 2017; published 1 September 2017)

\begin{abstract}
Two generic methods based on frequentism and Bayesianism are presented in this work aiming to adequately estimate decay lifetimes from measured data, while accounting for restricted observation times in the measurements. All the experimental scenarios that can possibly arise from the observation constraints are treated systematically and formulas are derived. The methods are then tested against the decay data of bare isomeric ${ }^{94 m} \mathrm{Ru}^{44+}$, which were measured using isochronous mass spectrometry with a timing detector at the CSRe in Lanzhou, China. Applying both methods in three distinct scenarios yields six different but consistent lifetime estimates. The deduced values are all in good agreement with a prediction based on the neutral-atom value modified to take the absence of internal conversion into account. Potential applications of such methods are discussed.
\end{abstract}

DOI: 10.1103/PhysRevC.96.034302

\section{INTRODUCTION}

The lifetime of a radioactive particle, whether an unstable nuclear ground state or a metastable excited state, is of great interest in nuclear physics, not only because it is one of the fundamental properties to help characterize such a quantum system, but also due to its referential value for an experimentalist to properly design an adequate setup for related measurements. Specifically, the lifetime quantifies the rate of the radioactive decay by which the parent particle transmutes to its daughter. When expressed as a formula, the decay probability of a single particle until some measuring time $t$ is

$$
F(t)=1-e^{-t / \tau}, \quad 0<t<\infty,
$$

where $\tau$ parameterizes the lifetime. Differentiating $F(t)$ with respect to $t$ leads to the probability density function

$$
f(t)=\frac{d F(t)}{d t}=\frac{e^{-t / \tau}}{\tau},
$$

which, as suggested by its name, means the decay probability at a particular moment.

Although the experimental techniques for lifetime measurements manifest great variety (see, e.g., Refs. [1-5]), probably a straightforward method in light of Eq. (2) would be to "reproduce" the density function with data and extract the lifetime. In other words, the lifetime is obtained from the leastsquares fitting of an exponential function to a histogram, which arises from sorting individually measured decay timestamps into corresponding time bins. Indeed, this idea has influenced the nuclear spectroscopists so profoundly that almost all the fitting-based methods can be shown emerging from it, whether

*cxc@impcas.ac.cn directly or indirectly. While it is widely applied with great success, it is nevertheless important to note that least-squares fittings may lead to unreasonable results when the data at hand are so sparse that a proper histogram cannot be formed no matter how the data are binned.

Schmidt et al. had therefore come up with an ingenious method to work with the sparse data by "squeezing" them in the logarithmic time scale [6]. Afterwards, a least-squares fitting could satisfactorily be applied. This mathematical trick was then widely adopted by nuclear spectroscopists, in particular for super-heavy-nuclei data analyses (see, e.g., Refs. [7-11]).

However, when applying the logarithmic-time-scale method, an assumption that the data are collected with no measuring time constraints, should be respected. This was also noted by Schmidt et al. in Ref. [6] with wording "... the observation time is not restricted ...". Obviously, such a condition can only be approximately satisfied in reality by extending the measuring time sufficiently (a quantitative definition is given in Sec. II A). Besides, in some occasional cases only the total number of decay events in a duration can at best be counted, for which the logarithmic-time-scale method is unfortunately not applicable. Hence, a more sophisticated data analysis scheme has to be developed to account for those complex experimental scenarios.

\section{FORMALISM}

Without loss of generality, the observation time is assumed to be restricted to the window $(0, T)$. Other intervals not starting at the origin can simply be shifted to 0 by redefining the timing reference. Equivalently, the stopping moment $T$ has divided the whole domain into two intervals, namely $(0, T)$ and $(T, \infty)$. For each interval, the experimental knowledge about the decay process in question can only take one of the following three options. 


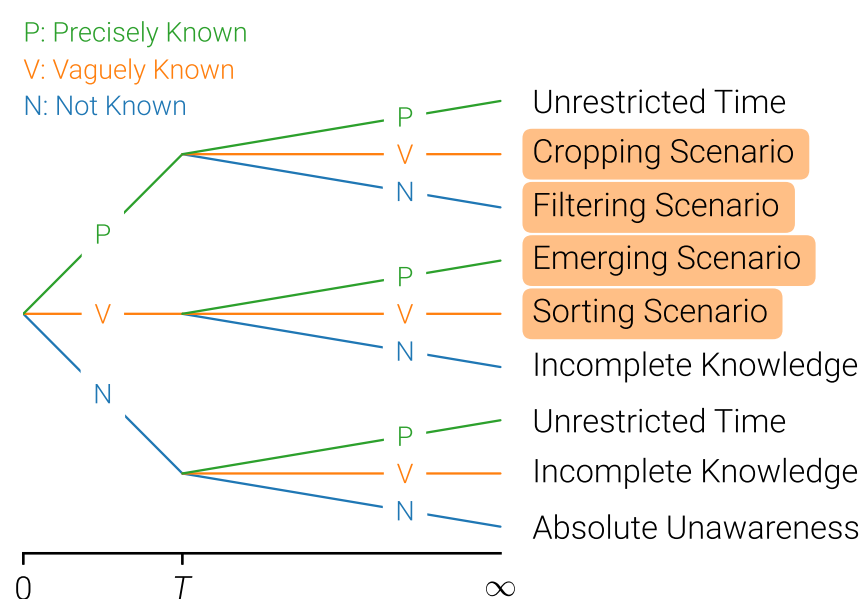

FIG. 1. Schematic presentation of nine possible scenarios of decay experiments when a restricted observation time is involved. The highlighted four with shade are the current scope of this work. See the text for details.

Precisely Known: Every decay time stamp is recorded.

Vaguely Known: Total decay number is counted.

Not Known: No decay quantities are measured.

Of nine possible scenarios shown schematically in Fig. 1, the $P-P$ pair trivially is the unrestricted-time scenario, which has been addressed by Schmidt et al. in Ref. [6]; the $N-P$ pair also represents the unrestricted time but with a shifted starting moment; the $V-N$ and $N-V$ pairs both lack information, such as the entire temporal span of the experiment, to evaluate the lifetime; the $N-N$ pair obviously contains no information. The rest of the four scenarios-namely filtering, cropping, emerging, and sorting — are to be treated in the following.

\section{A. Filtering scenario}

In the filtering scenario, $n$ decay events are observed in the time window $(0, T)$. Their occurring timestamps $\left\{t_{i}\right\}$ with $i$ running from 1 to $n$ are correspondingly measured. The window acts like a filter-only those timestamps falling into the range $(0, T)$ are selected.

Statistically, the decay probability $G(t)$ of a particle until some measuring time $t$ for the filtering scenario is in fact the conditional probability of $F(t)$

$$
G(t)=F(t \mid 0<t<T)=\frac{F(t)}{F(T)} .
$$

At a specific moment, the decay probability is given by the respective density function $g(t)$

$$
g(t)=\frac{d G(t)}{d t}=\frac{e^{-t / \tau}}{\tau\left(1-e^{-T / \tau}\right)}, \quad 0<t<T .
$$

By comparison of Eqs. (2) and (4), $g(t)$ is modified from $f(t)$ by a multiplicative factor $1 /\left(1-e^{-T / \tau}\right)$, which characterizes the degree of modification. Its dependency on the ratio $T / \tau$ is illustrated in Fig. 2. It is evident that the modified density $g(t)$ can well be approximated by $f(t)$ only if $T>3 \tau$. In other words, the window length should be three

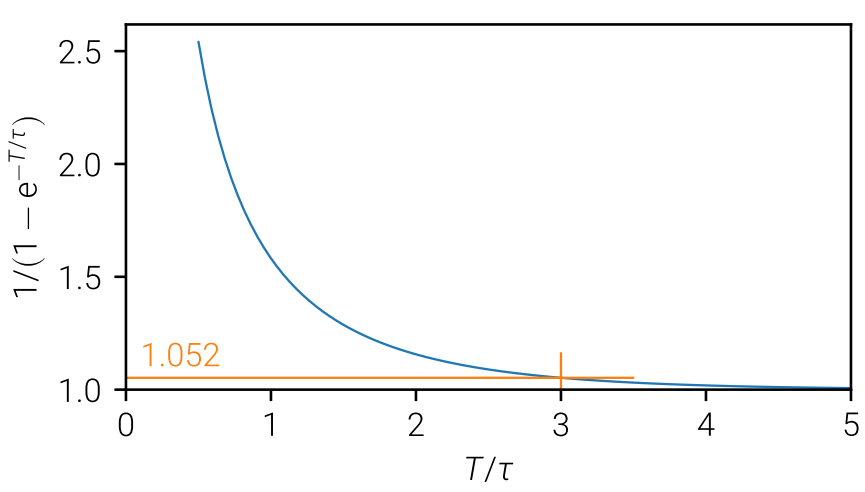

FIG. 2. Degree of modification as a function of the observation time. $g(t)$ is closer to $f(t)$ as the observation time gets longer.

times longer than the lifetime in question so as to assure the applicability of the logarithmic-time-scale method.

Based on Eq. (4), the mean $\mu$ and the variance $\sigma^{2}$ of $g(t)$ are obtained from integrals

$$
\begin{gathered}
\mu=E\{t\}=\int_{0}^{T} \operatorname{tg}(t) d t, \\
\sigma^{2}=\operatorname{Var}\{t\}=\int_{0}^{T} t^{2} g(t) d t-\mu^{2},
\end{gathered}
$$

which can be reduced to

$$
\begin{aligned}
\mu & =\tau\left(1-\frac{T / \tau}{e^{T / \tau}-1}\right), \\
\sigma^{2} & =\tau^{2}\left(1-\frac{(T / 2 \tau)^{2}}{\sinh ^{2}(T / 2 \tau)}\right) .
\end{aligned}
$$

Under the asymptotic condition $T \rightarrow \infty$, the special relations $\mu=\tau$ and $\sigma^{2}=\tau^{2}$ for $f(t)$ are restored.

\section{Frequentist inference}

Frequentist statistics regards every parameter in the estimating model to be a fixed quantity, which is the lifetime $\tau$ in the current case. Given a $\tau$, the measured decay timestamps are dictated by the probability density function $g(t)$ in Eq. (4). The likelihood function $L(\mathcal{D} \mid \tau)$ is then by definition written as

$$
L(\mathcal{D} \mid \tau)=\prod_{i=1}^{n} g\left(t_{i}\right)=\left(\frac{e^{-\bar{t} / \tau}}{\tau\left(1-e^{-T / \tau}\right)}\right)^{n},
$$

where $\mathcal{D}$ represents the data set, $\bar{t}=\left(\sum_{i=1}^{n} t_{i}\right) / n$ is the sample mean. It may be worth mentioning that the notation $L(\mathcal{D} \mid \tau)$ merely indicates a fixed $\tau$ with no implications on conditional probability whatsoever, in contrast to the interpretation adopted in Sec. II A 2.

By means of the maximum likelihood estimator, the point estimate $\hat{\tau}$ must satisfy the equation

$$
\left.\frac{\partial \ln L(\mathcal{D} \mid \tau)}{\partial \tau}\right|_{\tau=\hat{\tau}}=0,
$$


which can be simplified to

$$
\bar{t}=\hat{\tau}\left(1-\frac{T / \hat{\tau}}{e^{T / \hat{\tau}}-1}\right) .
$$

Comparing Eqs. (7) and (11), one finds that the maximum likelihood estimator is consistent with directly setting $\mu=\bar{t}$, which appears quite natural to one's intuition.

It is, however, important to note that the solution to Eq. (11) does not necessarily exist. This can be corroborated by investigating the behavior of the right side of Eq. (11)

$$
\theta(\tau)=\tau\left(1-\frac{T / \tau}{e^{T / \tau}-1}\right)
$$

Since $\theta(\tau)$ is monotonically increasing, it reaches its maximum only when $\tau \rightarrow \infty$, given that $\theta(\tau)$ is convergent. Indeed, by means of substitution $\lambda=1 / \tau$ and L'Hôpital's rule, it is found that the maximum is $T / 2$. Consequently, if $\bar{t}>T / 2$, which is, in principle, not to be taken for granted, Eq. (11) does not have a real root. In practice when this does occur, it can be a message to the experimentalist suggesting that whether the data are insufficient or the simplex decay model inadequately describes the particular physical process. On the contrary, as will be shown in Sec. II A 2, Bayesian inference still applies to the sparse data even down to single events.

If the maximum likelihood estimate $\hat{\tau}$ exists, it must satisfy the Cramér-Rao lower bound (see, e.g., Ref. [12]). In other words, the variance of $\hat{\tau}$ is given by the relation

$$
\operatorname{Var}\{\hat{\tau}\}=\frac{1}{I_{n}(\hat{\tau})},
$$

with $I_{n}(\tau)$ written as

$$
I_{n}(\tau)=\mathrm{E}\left\{\left(\frac{\partial \ln L(\mathcal{D} \mid \tau)}{\partial \tau}\right)^{2}\right\}
$$

By virtue of the identity

$$
\int_{0}^{T} \frac{\partial \ln g(t)}{\partial \tau} g(t) d t=\int_{0}^{T} \frac{\partial g(t)}{\partial \tau} d t=0,
$$

Eq. (14) can be simplified to

$$
\begin{aligned}
I_{n}(\tau) & =E\left\{\left(\sum_{i=1}^{n} \frac{\partial \ln g\left(t_{i}\right)}{\partial \tau}\right)^{2}\right\} \\
& =\sum_{i=1}^{n} E\left\{\left(\frac{\partial \ln g\left(t_{i}\right)}{\partial \tau}\right)^{2}\right\}=n I(\tau),
\end{aligned}
$$

where

$$
I(\tau)=\int_{0}^{T}\left(\frac{\partial \ln g(t)}{\partial \tau}\right)^{2} g(t) d t
$$

is called the Fisher information. Hence Eq. (13) can be reduced to

$$
\operatorname{Var}\{\hat{\tau}\}=\frac{1}{n I(\hat{\tau})}
$$

After going through some tedious derivations, the variance of $\hat{\tau}$ finally reads

$$
\operatorname{Var}\{\hat{\tau}\}=\frac{\hat{\tau}^{4}}{n \sigma^{2}(\hat{\tau})}
$$

\section{Bayesian inference}

That Bayesian statistics treats every parameter in a model as a random variable is the key factor distinguishing itself from frequentist statistics in terms of philosophical stand. It uses measured data to update a priori knowledge about the parameter, resulting in a posteriori information (see, e.g., Ref. [13]). Specifically, in the present case, the lifetime $\tau$ is assumed to obey a prior density $\pi(\tau)$ before any measurements. Given a particular $\tau$, the chance of the measurement outcomes $\mathcal{D}$ is governed by the likelihood function already expressed in Eq. (9). Here, the likelihood is to be understood as a conditional probability. The updated knowledge, which is quantified by the posterior density, reads

$$
\pi(\tau \mid \mathcal{D}) \propto L(\mathcal{D} \mid \tau) \pi(\tau) .
$$

To avoid introducing any artificial bias, a common measure is to adopt Jeffreys prior. Because of its invariance property under reparameterization, it is considered to be noninformative and objective. With the Fisher information already given in Eq. (17), Jeffreys prior is defined as

$$
\pi(\tau) \propto \sqrt{I(\tau)}
$$

which can further be reduced to

$$
\pi(\tau) \propto \frac{\sigma}{\tau^{2}} .
$$

The posterior density is then written as

$$
\pi(\tau \mid \mathcal{D})=A\left(\frac{e^{-\bar{t} / \tau}}{\tau\left(1-e^{-T / \tau}\right)}\right)^{n} \frac{\sigma}{\tau^{2}},
$$

with the normalization factor $A$ to be determined from the identity

$$
\int_{0}^{\infty} \pi(\tau \mid \mathcal{D}) d \tau=1
$$

where the lower limit of 0 is set from physical consideration.

Once the posterior density is obtained, the point estimate $\hat{\tau}$ can be defined according to a certain estimator. For example, the frequently adopted minimum-mean-square-error estimator will result in the posterior mean. Unfortunately, it does not apply in the present case, as the mean is nonexistent. This can heuristically be reasoned by investigating the asymptotic behavior of $\pi(\tau \mid \mathcal{D})$ when $\tau \rightarrow \infty$. By means of substitution $\lambda=1 / \tau$ then the Taylor expansion around 0 , the prior density in Eq. (22) behaves asymptotically like

$$
\pi(\tau) \sim \frac{1}{\tau^{2}}, \quad \tau \rightarrow \infty .
$$

The likelihood function in Eq. (9), on the other hand, converges as $\tau \rightarrow \infty$. In total, the posterior density shares the same behavior as of the prior density. (See also the inset of Fig. 3.) Despite that the posterior density is proper owing to the 


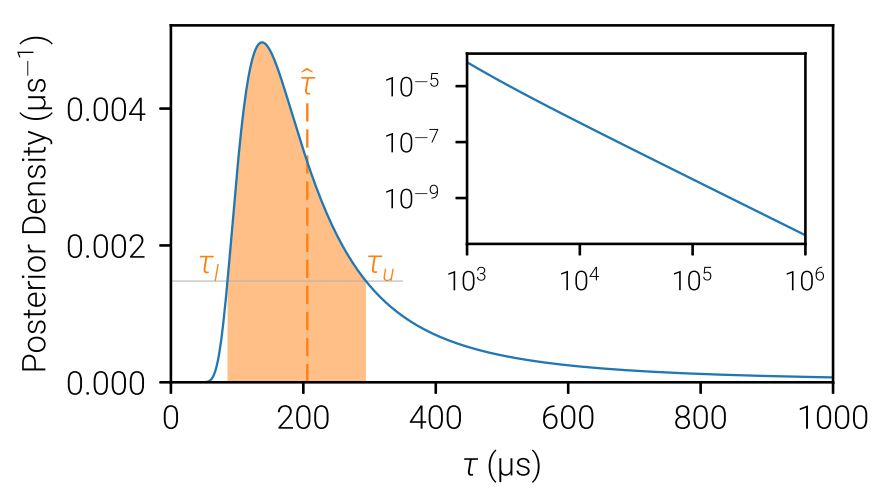

FIG. 3. Posterior density for the lifetime estimation of the experimental data in the filtering scenario from bare isomeric ${ }^{94 m} \mathrm{Ru}^{44+}$ decays in the laboratory frame. The dashed line indicates the point estimate, while the shaded region marks the credible interval at $68.3 \%$ level. The inset in log-log scale demonstrates the asymptotic inverse-square relationship on $\tau$.

inverse-square proportionality on $\tau$, its mean is ill defined since the integral of $\tau \pi(\tau \mid \mathcal{D})$ to $\infty$ diverges.

An alternative for the point estimate $\hat{\tau}$ can be the posterior median, which is also often used. By definition, $\hat{\tau}$ must satisfy the equation

$$
\int_{0}^{\hat{\imath}} \pi(\tau \mid \mathcal{D}) d \tau=0.5
$$

The "one-sigma" credible interval $\left(\tau_{l}, \tau_{u}\right)$, which is an analog to the confidence interval in frequentism, must satisfy

$$
\int_{\tau_{l}}^{\tau_{u}} \pi(\tau \mid \mathcal{D}) d \tau=68.3 \%
$$

An additional constraint by minimizing the distance $\tau_{u}-\tau_{l}$ is manually posed so as to fix the interval. By means of Lagrange multiplier, this condition is in fact equivalent to

$$
\pi\left(\tau_{l} \mid \mathcal{D}\right)=\pi\left(\tau_{u} \mid \mathcal{D}\right) .
$$

\section{B. Cropping scenario}

In the cropping scenario, apart from $n$ timestamps $\left\{t_{i}\right\}$ in the time window $(0, T)$ are measured, $m$ survived particles are also observed throughout the window. In other words, those $m$ decay timestamps falling outside the window are cropped to $T$.

At a particular measuring moment $t \in(0, T)$, the decay probability is dictated by $f(t)$ expressed in Eq. (2), while at $t=T$, it is quantified by the survival probability $S$

$$
S=\int_{T}^{\infty} f(t) d t=e^{-T / \tau} .
$$

Consequently, the total probability amounts to unity.

The likelihood function can similarly be constructed as

$$
\begin{aligned}
L(\mathcal{D} \mid \tau) & =\left(\begin{array}{c}
n+m \\
n
\end{array}\right) \prod_{i=1}^{n} f\left(t_{i}\right) S^{m} \\
& =\left(\begin{array}{c}
n+m \\
n
\end{array}\right)\left(\frac{e^{-\bar{t} / \tau}}{\tau}\right)^{n} e^{-m T / \tau} .
\end{aligned}
$$

According to the maximum likelihood estimator, the point estimate $\hat{\tau}$ in the frequentist context simply reads

$$
\hat{\tau}=\bar{t}+\frac{m T}{n} .
$$

Note that Eq. (31) is essentially the method used by Gray et al., although without proof, for the lifetime estimation in Ref. [14]. Again the variance of $\hat{\tau}$ is obtained by calculating the CramérRao lower bound, which becomes complicated in the current case due to the mixed random variable $t$ of continuous and discrete types. Accordingly, the Fisher information in Eq. (17) is generalized to a mixture of integration and addition

$$
\begin{aligned}
I(\tau) & =\int_{0}^{T}\left(\frac{\partial \ln f(t)}{\partial \tau}\right)^{2} f(t) d t+\left(\frac{\partial \ln S}{\partial \tau}\right)^{2} S \\
& =\frac{1-e^{-T / \tau}}{\tau^{2}},
\end{aligned}
$$

which then gives rise to

$$
\operatorname{Var}\{\hat{\tau}\}=\frac{\hat{\tau}^{2}}{(n+m)\left(1-e^{-T / \hat{\tau}}\right)} .
$$

With the newly obtained Fisher information in Eq. (32), the prior density in the Bayesian context reads

$$
\pi(\tau) \propto \frac{\sqrt{1-e^{-T / \tau}}}{\tau} .
$$

Combing Eqs. (30) and (34) leads to the posterior density

$$
\begin{aligned}
\pi(\tau \mid \mathcal{D}) \propto & \left(\begin{array}{c}
n+m \\
n
\end{array}\right)\left(\frac{e^{-\bar{t} / \tau}}{\tau}\right)^{n} e^{-m T / \tau} \\
& \times \frac{\sqrt{1-e^{-T / \tau}}}{\tau} .
\end{aligned}
$$

By inspecting the right side of Eq. (35), it is found that $\pi(\tau \mid \mathcal{D})$ is continuous in the full domain $(0, \infty)$ with zero at $\tau=0$. Its asymptotic behavior when $\tau \rightarrow \infty$ is

$$
\pi(\tau \mid \mathcal{D}) \sim \frac{1}{\tau^{n+3 / 2}}, \quad \tau \rightarrow \infty,
$$

of which the mean always exists owing to $n \geqslant 1$. By virtue of the minimum-mean-square-error estimator, the point estimate $\hat{\tau}$ is

$$
\hat{\tau}=\int_{0}^{\infty} \tau \pi(\tau \mid \mathcal{D}) d \tau,
$$

with the variance given as

$$
\operatorname{Var}\{\tau\}=\int_{0}^{\infty} \tau^{2} \pi(\tau \mid \mathcal{D}) d \tau-\hat{\tau}^{2} .
$$

Note that the variance is undefined when $n=1$. The "onesigma" credible interval should instead be resorted to in this particular case, as already demonstrated in Sec. II A 2.

\section{Emerging scenario}

In the emerging scenario, $n$ particles are known to be dead prior to the measurement starting at $T$. Then $m$ decay events are observed, their corresponding timestamps $\left\{t_{j}\right\}$ with $j$ running from 1 to $m$ are measured. Those decay timestamps are said to 
emerge from the detection limit $T$. In principle, the observation time ought to extend to infinity. Pragmatically, according to the criterion in Sec. II A, the observation time should be at least three times longer than the lifetime to be measured. In what follows, this requirement is assumed to be fulfilled.

Similar to the cropping scenario, the decay probability is modeled by a mixed distribution. The random variable $t$ is discrete at $t=T$ with the dead probability given as

$$
D=\int_{0}^{T} f(t) d t=1-e^{-T / \tau},
$$

whereas $t$ is continuous in $(T, \infty)$ with the probability density $f(t)$ given in Eq. (2).

The likelihood function now becomes

$$
\begin{aligned}
L(\mathcal{D} \mid \tau) & =\left(\begin{array}{c}
n+m \\
n
\end{array}\right) D^{n} \prod_{j=1}^{m} f\left(t_{j}\right) \\
& =\left(\begin{array}{c}
n+m \\
n
\end{array}\right)\left(1-e^{-T / \tau}\right)^{n}\left(\frac{e^{-\bar{t} / \tau}}{\tau}\right)^{m},
\end{aligned}
$$

where the sample mean $\bar{t}=\left(\sum_{j=1}^{m} t_{j}\right) / m$ is substituted. The frequentist inference by means of the maximum likelihood estimator leads to the point estimate $\hat{\tau}$ determined from the relation

$$
\bar{t}=\hat{\tau}+\frac{n T}{m\left(e^{T / \hat{\tau}}-1\right)} .
$$

In a similar vein to Eq. (32), Fisher information is expressed as

$$
\begin{aligned}
I(\tau) & =\left(\frac{\partial \ln D}{\partial \tau}\right)^{2} D+\int_{T}^{\infty}\left(\frac{\partial \ln f(t)}{\partial \tau}\right)^{2} f(t) d t \\
& =\frac{1}{\tau^{4}}\left(\frac{T^{2}}{e^{T / \tau}-1}+\frac{\tau^{2}}{e^{T / \tau}}\right)
\end{aligned}
$$

Hence the variance of $\hat{\tau}$ reads

$$
\operatorname{Var}\{\hat{\tau}\}=\frac{\hat{\tau}^{4}}{n+m} /\left(\frac{T^{2}}{e^{T / \hat{\tau}}-1}+\frac{\hat{\tau}^{2}}{e^{T / \hat{\imath}}}\right) .
$$

For the Bayesian inference, the prior density is given as

$$
\pi(\tau) \propto \frac{1}{\tau^{2}} \sqrt{\frac{T^{2}}{e^{T / \tau}-1}+\frac{\tau^{2}}{e^{T / \tau}}}
$$

which, together with Eq. (40), leads to the posterior density

$$
\begin{aligned}
\pi(\tau \mid \mathcal{D}) \propto & \left(\begin{array}{c}
n+m \\
n
\end{array}\right)\left(1-e^{-T / \tau}\right)^{n}\left(\frac{e^{-\bar{t} / \tau}}{\tau}\right)^{m} \\
& \times \frac{1}{\tau^{2}} \sqrt{\frac{T^{2}}{e^{T / \tau}-1}+\frac{\tau^{2}}{e^{T / \tau}}}
\end{aligned}
$$

It can be found that $\pi(\tau \mid \mathcal{D})$ is continuous in $(0, \infty)$ with zero at $\tau=0$. Its asymptotic behavior when $\tau \rightarrow \infty$ is

$$
\pi(\tau \mid \mathcal{D}) \sim \frac{1}{\tau^{n+m+1}}, \quad \tau \rightarrow \infty,
$$

of which the mean certainly exists due to $n \geqslant 1$ and $m \geqslant 1$. The point estimate $\hat{\tau}$ thus reads

$$
\hat{\tau}=\int_{0}^{\infty} \tau \pi(\tau \mid \mathcal{D}) d \tau
$$

with the variance given as

$$
\operatorname{Var}\{\tau\}=\int_{0}^{\infty} \tau^{2} \pi(\tau \mid \mathcal{D}) d \tau-\hat{\tau}^{2} .
$$

Specifically, if $n=m=1$, the integral in Eq. (48) diverges, hence the "one-sigma" credible interval should be used instead.

\section{Sorting scenario}

In the sorting scenario, $n$ decay events are observed in the time window $(0, T)$, while $m$ particles survive throughout the window. However, no explicit timestamps are measured. All the decay events are sorted into two contiguous intervals separated by $T$.

This scenario is essentially $n+m$ times successive Bernoulli trials with a dead probability given in Eq. (39) and its complementary survival probability given in Eq. (29). The likelihood function is consequently written as

$$
\begin{aligned}
L(\mathcal{D} \mid \tau) & =\left(\begin{array}{c}
n+m \\
n
\end{array}\right) D^{n} S^{m} \\
& =\left(\begin{array}{c}
n+m \\
n
\end{array}\right)\left(1-e^{-T / \tau}\right)^{n} e^{-m T / \tau} .
\end{aligned}
$$

The point estimate $\hat{\tau}$ according to the maximum likelihood estimator is obtained to be

$$
\hat{\tau}=\frac{T}{\ln (1+n / m)} .
$$

The Fisher information in the present case should be written as

$$
\begin{aligned}
I(\tau) & =\left(\frac{\partial \ln D}{\partial \tau}\right)^{2} D+\left(\frac{\partial \ln S}{\partial \tau}\right)^{2} S \\
& =\frac{T^{2}}{\tau^{4}\left(e^{T / \tau}-1\right)}
\end{aligned}
$$

Thus the variance of $\hat{\tau}$ accordingly reads

$$
\operatorname{Var}\{\hat{\tau}\}=\frac{\hat{\tau}^{4}\left(e^{T / \hat{\tau}}-1\right)}{(n+m) T^{2}} .
$$

For the Bayesian inference, the prior density is written as

$$
\pi(\tau) \propto \frac{T}{\tau^{2} \sqrt{e^{T / \tau}-1}},
$$

while the posterior density reads

$$
\begin{aligned}
\pi(\tau \mid \mathcal{D}) \propto & \left(\begin{array}{c}
n+m \\
n
\end{array}\right)\left(1-e^{-T / \tau}\right)^{n} e^{-m T / \tau} \\
& \times \frac{T}{\tau^{2} \sqrt{e^{T / \tau}-1}} .
\end{aligned}
$$


Similarly, $\pi(\tau \mid \mathcal{D})$ is continuous in $(0, \infty)$ with zero at $\tau=0$. Its asymptotic behavior when $\tau \rightarrow \infty$ is

$$
\pi(\tau \mid \mathcal{D}) \sim \frac{1}{\tau^{n+3 / 2}}, \quad \tau \rightarrow \infty,
$$

of which the mean always exists owing to $n \geqslant 1$. Consequently the point estimate in the Bayesian context is given as

$$
\hat{\tau}=\int_{0}^{\infty} \tau \pi(\tau \mid \mathcal{D}) d \tau,
$$

with the variance

$$
\operatorname{Var}\{\tau\}=\int_{0}^{\infty} \tau^{2} \pi(\tau \mid \mathcal{D}) d \tau-\hat{\tau}^{2} .
$$

In particular when $n=1$, the nonexistent variance should be replaced by the "one-sigma" credible interval.

\section{CASE STUDY: LIFETIME OF ${ }^{94 m} \mathbf{R u}^{44+}$}

In an experiment conducted at the experimental Cooler Storage Ring (CSRe) in Lanzhou, China, bare ${ }^{94} \mathrm{Ru}^{44+}$ ions in nuclear ground and isomeric $\left(2644 \mathrm{keV}, 8^{+}\right)$states were measured when the ring is tuned to an isochronous mode. The transition point for the ion-optical setting was 1.302, which is equal to the Lorentz factor of target ions. The lap times of the circulating ions at each revolution were registered by a timing detector at a fixed location in the ring. Individual decays of the isomeric ${ }^{94 m} \mathrm{Ru}^{44+}$, which were signaled by abrupt changes in lap times due to the mass difference between ground and isomeric states, were unambiguously identified in a restricted observation time window from 20 to $180 \mu \mathrm{s}$. Owing to the instrumental limits, the decay timestamps of any events that occurred outside the window could not be determined accurately, and thus were neglected. (In fact, only two candidates were discarded.) In total, 49 valid decay events were observed in that experiment. The corresponding timestamps led to a sample mean of $90.3 \mu \mathrm{s}$. For more details on the experiment setup and timestamps extraction, the reader is referred to Ref. [15].

The measurement condition in that experiment agrees with the filtering scenario, except for the time window starting at $20 \mu \mathrm{s}$. By redefining the timing reference, the window essentially is $(0,160) \mu \mathrm{s}$, while the sample mean is reduced to $70.3 \mu$ s. With the values $T=160, \bar{t}=70.3, n=49$, Eqs. (11) and (19) give rise to the lifetime estimate of $217.5 \pm 148.3 \mu \mathrm{s}$ in frequentism. On the other hand, the posterior density for Bayesian inference is plotted in Fig. 3. By using Eqs. (26), (27), and (28), the posterior density leads to the point estimate of $206.0 \mu \mathrm{s}$ within the credible interval of $(85.5,294.4) \mu \mathrm{s}$.

In the same experiment, the atomic masses of ${ }^{94} \mathrm{Ru}$ in the nuclear ground and isomeric states were also measured with the isochronous mass spectrometry. If integrating the results from mass measurements, an additional 29 isomers were identified to survive throughout the observation window. However, a parasitic effect of the data analysis for the mass determination is to deselect those injections with deficient calibrating nuclides [16]. This process decreased

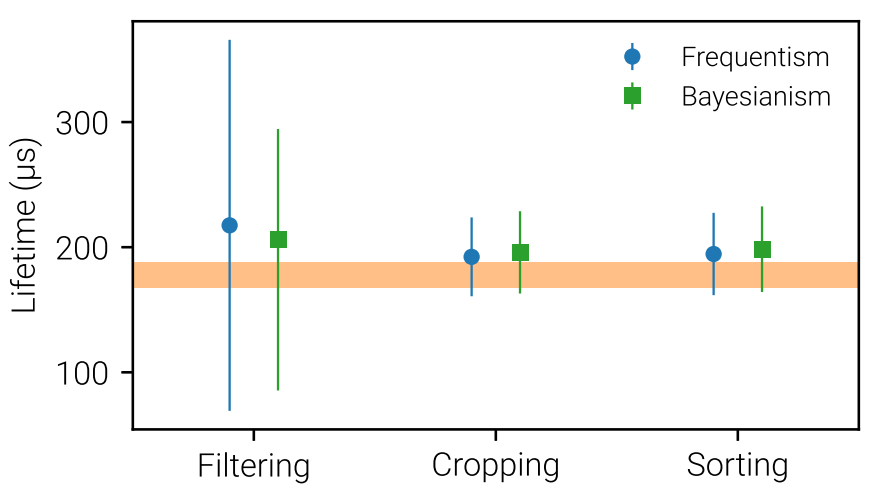

FIG. 4. Comparison between six lifetime estimates for the bare isomeric ${ }^{94 m} \mathrm{Ru}^{44+}$ in the laboratory frame by means of frequentist and Bayesian inferences in three distinct experimental scenarios. The horizontal band indicates the theoretical prediction range.

the former 49 decays to 37 decays whose occurring timestamps were explicitly measured. The sample mean now becomes $86.9 \mu \mathrm{s}$.

Accordingly, this is the cropping experimental scenario, with $T=160, \bar{t}=66.9, n=37, m=29$. The lifetime estimate from frequentist inference is obtained to be $192.3 \pm$ $31.5 \mu$ s by substituting those values into Eqs. (31) and (33). Similarly, by virtue of Eqs. (37) and (38), Bayesian inference gives rise to $195.9 \pm 32.9 \mu \mathrm{s}$.

Moreover, the sorting scenario can also be tested against the data if the decay timestamps are intentionally ignored. Thus, the selected $T=160, n=37, m=29$ are used in Eqs. (50) and (52) for the frequentist inference, which results in $194.6 \pm$ $32.9 \mu \mathrm{s}$ as the lifetime estimate. The Bayesian inference, on the other hand, yields $198.4 \pm 34.2 \mu$ s using Eqs. (56) and (57).

The literature value for the half-life of isomeric ${ }^{94 m} \mathrm{Ru}$ as neutral atoms at rest is $71 \pm 4 \mu$ s [17]. They decay predominantly via isomeric transition to the $6^{+}$state which lies $146 \mathrm{keV}$ lower in energy, whereas other competing processes are negligible [18]. Once the atoms are fully stripped, the decay channel by internal conversion is consequently shut off. The bare isomers can only lose energy via photon emission. By means of the internal conversion coefficient of 0.335 computed with the BRICC code [19], the theoretical prediction for the boosted lifetime of ${ }^{94 m} \mathrm{Ru}^{44+}$ in the laboratory frame $(\gamma=1.302)$ is $178 \pm 10 \mu$ s.

Figure 4 shows a pictorial comparison between the aforementioned lifetime estimates together with the predicted value. In spite of the larger error margin associated with the filtering scenario, which can be attributed to the smaller sample size, all the estimates are consistent with each other and exhibit good agreement with the prediction. Moreover, the Bayesian inference yields a tighter uncertainty range than the frequentist inference in the filtering scenario, otherwise they both lead to quite similar estimates. Note that the resultant estimates are comparable to the length of the observation window, which, in turn, supports the choice of the present methods over the logarithmic-time-scale method. Indeed, a deficient estimate 


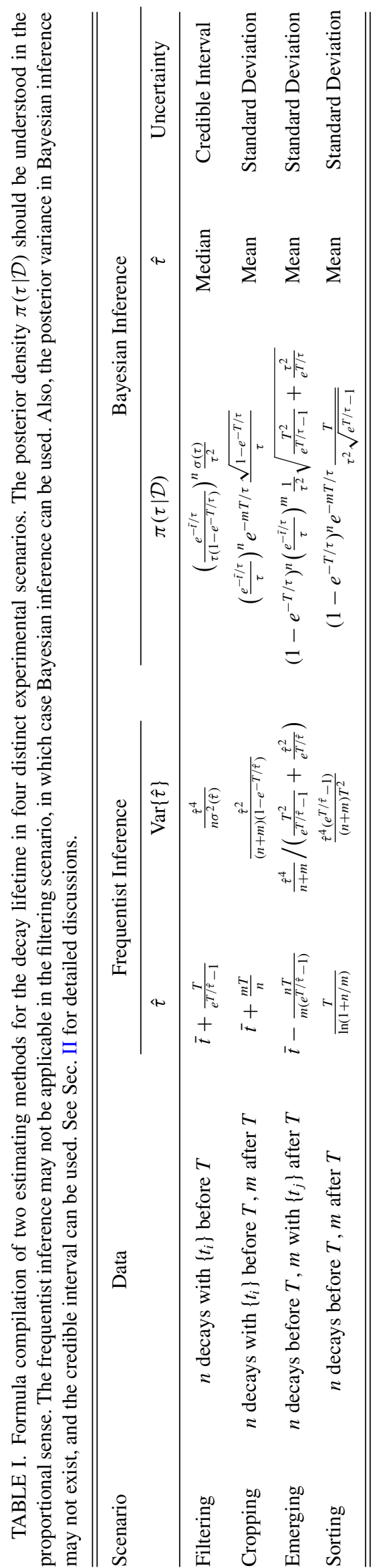

of $92 \pm 16 \mu$ s is erroneously obtained by means of this last method.

\section{CONCLUSION AND OUTLOOK}

Owing to the various practical constraints, the observation time for a decay process is generally restricted. The experimental scenarios thus become complex with such a restriction involved. The applicability of conventional data analysis methods for the lifetime evaluation faces great challenges, in particular when the available data manifest scarcity. Out of nine categorized scenarios, four of special interest-i.e., filtering, cropping, emerging, and sorting scenarios-were described and investigated systematically in this work. Two generic statistics-based methods were presented to properly estimate the lifetime in those four scenarios. The resultant formulas are compiled in Table I for easy reference. With the methods being successfully tested in the filtering, cropping, and sorting scenarios, their validity in the emerging scenario is assured. Even though the temporal restriction virtually disappears when the observation time is three times longer than the lifetime, it is strongly suggested to use the methods in this work to analyze decay data, especially for any lifetimes not known beforehand, to avoid any systematic errors.

Possible applications of the presented methods include, for instance, ring-based $\beta$-decay studies with beam cooling applied to extend the storage time [20]. Experiments of such a kind have routinely been conducted in the ESR at GSI, Germany for decades [21-25]. With the intensity-sensitive and time-resolving Schottky resonator recently installed in the ESR [26], decays of exotic ions can be investigated on a single-ion basis $[27,28]$. The fates of stored ions can be traced individually within the observation time of typically several minutes, which is, in fact, the cropping scenario. At the CSRe in Lanzhou, a similar experimental campaign is envisaged in the near future. One interesting physics case is to compare the lifetimes of bare and hydrogen-like ${ }^{111} \mathrm{Sn}$ ions to verify the angular momentum selection rule in electron capture decay [29]. The sorting scenario can be realized in the rare-RI ring at RIKEN, Japan, although lifetime measurements seem not be the main motivation for the construction of the ring [30]. When placing two detection systems one at the entrance and the other at the exit of the ring for the respective identification of mother and daughter ions, the number of decay events during the storage can be counted, from which the decay lifetime can be deduced.

\section{ACKNOWLEDGMENTS}

This work was partially supported by the Chinese National Key Program for S\&T Research and Development (No. 2016YFA0400504), by the Major State Basic Research Development Program of China (No. 2013CB834401), and by the National Natural Science Foundation of China (No. U1232208, No. U1432125). X.L.T. acknowledges supports by the Chinese Academy of Sciences Pioneer Hundred Talents Program, and by the Max-Plank-Society. Y.A.L. acknowledges the support by the Chinese Academy of Sciences President's International Fellowship Initiative (No. 2016VMA043). P.M.W. is supported by the UK Science and Technology Facilities Council. 
[1] T. Ohtsuki, H. Yuki, M. Muto, J. Kasagi, and K. Ohno, Enhanced Electron-Capture Decay Rate of ${ }^{7} \mathrm{Be}$ Encapsulated in $\mathrm{C}_{60}$ Cages, Phys. Rev. Lett. 93, 112501 (2004).

[2] L. J. Broussard, H. O. Back, M. S. Boswell, A. S. Crowell, P. Dendooven, G. S. Giri, C. R. Howell, M. F. Kidd, K. Jungmann, W. L. Kruithof, A. Mol, C. J. G. Onderwater, R. W. Pattie, P. D. Shidling, M. Sohani, D. J. van der Hoek, A. Rogachevskiy, E. Traykov, O. O. Versolato, L. Willmann, H. W. Wilschut, and A. R. Young, Measurement of the Half-Life of the $T=\frac{1}{2}$ Mirror Decay of ${ }^{19} \mathrm{Ne}$ and its Implication on Physics Beyond the Standard Model, Phys. Rev. Lett. 112, 212301 (2014).

[3] A. Lennarz, A. Grossheim, K. G. Leach, M. Alanssari, T. Brunner, A. Chaudhuri, U. Chowdhury, J. R. Crespo LópezUrrutia, A. T. Gallant, M. Holl, A. A. Kwiatkowski, J. Lassen, T. D. Macdonald, B. E. Schultz, S. Seeraji, M. C. Simon, C. Andreoiu, J. Dilling, and D. Frekers, In-Trap Spectroscopy of Charge-Bred Radioactive Ions, Phys. Rev. Lett. 113, 082502 (2014).

[4] R. N. Wolf, D. Atanasov, K. Blaum, S. Kreim, D. Lunney, V. Manea, M. Rosenbusch, L. Schweikhard, A. Welker, F. Wienholtz, and K. Zuber, Background-free beta-decay half-life measurements by in-trap decay and high-resolution MR-ToF mass analysis, Nucl. Instrum. Meth. B 376, 275 (2016).

[5] B. Seiferle, L. von der Wense, and P. G. Thirolf, Lifetime Measurement of the ${ }^{229}$ Th Nuclear Isomer, Phys. Rev. Lett. 118, 042501 (2017).

[6] K. H. Schmidt, C. C. Sahm, K. Pielenz, and H. G. Clerc, Some remarks on the error analysis in the case of poor statistics, $\mathrm{Z}$. Phys. A 316, 19 (1984).

[7] Y. T. Oganessian, A. V. Yeremin, A. G. Popeko, S. L. Bogomolov, G. V. Buklanov, M. L. Chelnokov, V. I. Chepigin, B. N. Gikal, V. A. Gorshkov, G. G. Gulbekian, M. G. Itkis, A. P. Kabachenko, A. Y. Lavrentev, O. N. Malyshev, J. Rohac, R. N. Sagaidak, S. Hofmann, S. Saro, G. Giardina, and K. Morita, Synthesis of nuclei of the superheavy element 114 in reactions induced by ${ }^{48} \mathrm{Ca}$, Nature 400, 242 (1999).

[8] Yu. Ts. Oganessian, F. Sh. Abdullin, P. D. Bailey, D. E. Benker, M. E. Bennett, S. N. Dmitriev, J. G. Ezold, J. H. Hamilton, R. A. Henderson, M. G. Itkis, Yu. V. Lobanov, A. N. Mezentsev, K. J. Moody, S. L. Nelson, A. N. Polyakov, C. E. Porter, A. V. Ramayya, F. D. Riley, J. B. Roberto, M. A. Ryabinin, K. P. Rykaczewski, R. N. Sagaidak, D. A. Shaughnessy, I. V. Shirokovsky, M. A. Stoyer, V. G. Subbotin, R. Sudowe, A. M. Sukhov, Yu. S. Tsyganov, V. K. Utyonkov, A. A. Voinov, G. K. Vostokin, and P. A. Wilk, Synthesis of a New Element with Atomic Number $Z=117$, Phys. Rev. Lett. 104, 142502 (2010).

[9] L. Stavsetra, K. E. Gregorich, J. Dvorak, P. A. Ellison, I. Dragojevic, M. A. Garcia, and H. Nitsche, Independent Verification of Element 114 Production in the ${ }^{48} \mathrm{Ca}+{ }^{242} \mathrm{Pu}$ Reaction, Phys. Rev. Lett. 103, 132502 (2009).

[10] J. Dvorak, W. Brüchle, M. Chelnokov, R. Dressler, Ch. E. Düllmann, K. Eberhardt, V. Gorshkov, E. Jäger, R. Krücken, A. Kuznetsov, Y. Nagame, F. Nebel, Z. Novackova, Z. Qin, M. Schädel, B. Schausten, E. Schimpf, A. Semchenkov, P. Thörle, A. Türler, M. Wegrzecki, B. Wierczinski, A. Yakushev, and A. Yeremin, Doubly Magic Nucleus ${ }_{108}^{270} \mathrm{Hs}_{162}$, Phys. Rev. Lett. 97, 242501 (2006).

[11] J. Khuyagbaatar et al., ${ }^{48} \mathrm{Ca}+{ }^{249} \mathrm{Bk}$ Fusion Reaction Leading to Element $Z=117$ : Long-Lived $\alpha$-Decaying ${ }^{270} \mathrm{Db}$ and Discovery of ${ }^{266}$ Lr, Phys. Rev. Lett. 112, 172501 (2014).
[12] A. Papoulis and S. U. Pillai, Probability, Random Variables and Stochastic Processes, 4th ed., (McGraw-Hill Europe, Boston, 2002).

[13] C. P. Robert, The Bayesian Choice: From Decision-Theoretic Foundations to Computational Implementation, 2nd ed., (Springer, New York, 2007).

[14] T. J. Gray, M. W. Reed, G. J. Lane, A. Akber, Yu. A. Litvinov, and P. M. Walker, Nuclear lifetime measurements from data with independently varying observation times, Eur. Phys. J. Conf. 123, 04004 (2016).

[15] Q. Zeng et al., Half-life measurement of short-lived ${ }_{44}^{94 m} \mathrm{Ru}^{44+}$ using isochronous mass spectrometry (unpublished).

[16] P. Shuai et al., Accurate correction of magnetic field instabilities for high-resolution isochronous mass measurements in storage rings, arXiv:1407.3459.

[17] G. Audi, F. G. Kondev, M. Wang, W. J. Huang, and S. Naimi, The NUBASE2016 evaluation of nuclear properties, Chin. Phys. C 41, 030001 (2017).

[18] D. Abriola and A. A. Sonzogni, Nuclear data sheets for $A=94$, Nucl. Data Sheets 107, 2423 (2006).

[19] T. Kibdi, T. W. Burrows, M. B. Trzhaskovskaya, P. M. Davidson, and C. W. Nestor, Jr., Evaluation of theoretical conversion coefficients using BrIcc, Nucl. Instrum. Meth. A 589, 202 (2008).

[20] Yu. A. Litvinov and F. Bosch, Beta decay of highly charged ions, Rep. Prog. Phys. 74, 016301 (2011).

[21] M. Jung, F. Bosch, K. Beckert, H. Eickhoff, H. Folger, B. Franzke, A. Gruber, P. Kienle, O. Klepper, W. Koenig, C. Kozhuharov, R. Mann, R. Moshammer, F. Nolden, U. Schaaf, G. Soff, P. Spädtke, M. Steck, Th. Stöhlker, and K. Sümmerer, First Observation of Bound-State $\beta^{-}$Decay, Phys. Rev. Lett. 69, 2164 (1992).

[22] H. Irnich, H. Geissel, F. Nolden, K. Beckert, F. Bosch, H. Eickhoff, B. Franzke, Y. Fujita, M. Hausmann, H. C. Jung, O. Klepper, C. Kozhuharov, G. Kraus, A. Magel, G. Münzenberg, F. Nickel, T. Radon, H. Reich, B. Schlitt, W. Schwab, M. Steck, K. Sümmerer, T. Suzuki, and H. Wollnik, Half-life Measurements of Bare, Mass-Resolved Isomers in a Storage-Cooler Ring, Phys. Rev. Lett. 75, 4182 (1995).

[23] F. Bosch, T. Faestermann, J. Friese, F. Heine, P. Kienle, E. Wefers, K. Zeitelhack, K. Beckert, B. Franzke, O. Klepper, C. Kozhuharov, G. Menzel, R. Moshammer, F. Nolden, H. Reich, B. Schlitt, M. Steck, T. Stöhlker, T. Winkler, and K. Takahashi, Observation of Bound-State $\beta^{-}$Decay of Fully Ionized ${ }^{187} \mathrm{Re}:{ }^{187} \mathrm{Re}^{187}$ Os Cosmochronometry, Phys. Rev. Lett. 77, 5190 (1996).

[24] T. Ohtsubo, F. Bosch, H. Geissel, L. Maier, C. Scheidenberger, F. Attallah, K. Beckert, P. Beller, D. Boutin, T. Faestermann, B. Franczak, B. Franzke, M. Hausmann, M. Hellström, E. Kaza, P. Kienle, O. Klepper, H.-J. Kluge, C. Kozhuharov, Yu. A. Litvinov, M. Matos, G. Münzenberg, F. Nolden, Yu. N. Novikov, M. Portillo, T. Radon, J. Stadlmann, M. Steck, T. Stöhlker, K. Sümmerer, K. Takahashi, H. Weick, M. Winkler, and T. Yamaguchi, Simultaneous Measurement of $\beta^{-}$Decay to Bound and Continuum Electron States, Phys. Rev. Lett. 95, 052501 (2005).

[25] Yu. A. Litvinov, F. Bosch, H. Geissel, J. Kurcewicz, Z. Patyk, N. Winckler, L. Batist, K. Beckert, D. Boutin, C. Brandau, L. Chen, C. Dimopoulou, B. Fabian, T. Faestermann, A. Fragner, L. Grigorenko, E. Haettner, S. Hess, P. Kienle, R. Knöbel, C. Kozhuharov, S. A. Litvinov, L. Maier, M. Mazzocco, F. Montes, 
G. Münzenberg, A. Musumarra, C. Nociforo, F. Nolden, M. Pfützner, W. R. Plaß, A. Prochazka, R. Reda, R. Reuschl, C. Scheidenberger, M. Steck, T. Stöhlker, S. Torilov, M. Trassinelli, B. Sun, H. Weick, and M. Winkler, Measurement of the $\beta^{+}$ and Orbital Electron-Capture Decay Rates in Fully Ionized, Hydrogenlike, and Heliumlike ${ }^{140}$ Pr Ions, Phys. Rev. Lett. 99, 262501 (2007).

[26] F. Nolden, P. Hülsmann, Yu. A. Litvinov, P. Moritz, C. Peschke, P. Petri, M. S. Sanjari, M. Steck, H. Weick, J. X. Wu, Y. D. Zang, S. H. Zhang, and T. C. Zhao, A fast and sensitive resonant Schottky pick-up for heavy ion storage rings, Nucl. Instrum. Meth. A 659, 69 (2011).

[27] Yu. A. Litvinov, F. Bosch, N. Winckler, D. Boutin, H. G. Essel, T. Faestermann, H. Geissel, S. Hess, P. Kienle, R. Knöbel, C. Kozhuharov, J. Kurcewicz, L. Maier, K. Beckert, P. Beller, C. Brandau, L. Chen, C. Dimopoulou, B. Fabian, A. Fragner,
E. Haettner, M. Hausmann, S.A. Litvinov, M. Mazzocco, F. Montes, A. Musumarra, C. Nociforo, F. Nolden, W. Plaß, A. Prochazka, R. Reda, R. Reuschl, C. Scheidenberger, M. Steck, T. Stöhlker, S. Torilov, M. Trassinelli, B. Sun, H. Weick, and M. Winkler, Observation of non-exponential orbital electron capture decays of hydrogen-like ${ }^{140} \mathrm{Pr}$ and ${ }^{142} \mathrm{Pm}$ ions, Phys. Lett. B 664, 162 (2008).

[28] P. Kienle et al., Two-Body-Weak-Decays Collaboration, Highresolution measurement of the time-modulated orbital electron capture and of the $\beta^{+}$decay of hydrogen-like ${ }^{142} \mathrm{Pm}^{60+}$ ions, Phys. Lett. B 726, 638 (2013).

[29] Yu. A. Litvinov, Mass and lifetime measurements at the present ESR facility, Int. J. Mod. Phys. E 18, 323 (2009).

[30] A. Ozawa, T. Uesaka, M. Wakasugi, and the Rare-RI Ring Collaboration, The rare-RI ring, Prog. Theor. Exp. Phys. 2012, 03C009 (2012). 